\title{
CURRENT MANAGEMENT OF ESTABLISHED DIABETIC EYE DISEASE
}

\author{
D. W. FLANAGAN \\ Cambridge
}

\begin{abstract}
SUMMARY
Diabetic patients with established diabetic eye disease are at risk of visual loss from vitreous haemorrhage, traction retinal detachment, macular oedema, cataract and eventually posterior capsule opacification. If there is an effective screening service, timely adequate photocoagulation should minimise visual loss from vitreous haemorrhage, traction retinal detachment and macular oedema. Vitreoretinal surgery should only be required in exceptional cases. Extracapsular cataract surgery and intraocular lens insertion can be followed by a dramatic worsening of pre-existing diabetic macular oedema. This can be prevented provided the severity of the retinopathy is recognised pre-operatively and treated appropriately by photocoagulation either before surgery or shortly afterwards. Neovascular glaucoma can follow YAG laser capsulotomy if the retina is very ischaemic. This likewise can be prevented provided the severity of the retinopathy is recognised and treated adequately by scatter photocoagulation.
\end{abstract}

The nature of diabetic eye disease, which is a major public health problem and important cause of severe visual handicap, has changed in the past 10 years. In the early 1980s many diabetics with what are called 'high risk characteristics' of disc neovascularisation and vitreous haemorrhage were not receiving adequate timely photocoagulation. As a result many young insulin-dependent (type 1) diabetics developed advanced diabetic eye disease with vitreous haemorrhage and traction retinal detachment. Complex vitreoretinal surgery offered the only hope of restoring limited vision to such patients. At that time the beneficial effect of early photocoagulation in the treatment of diabetic macular oedema was not widely appreciated, so many older non-insulin-dependent (type 2) diabetics lost central vision. In the past 10 years there has been a marked increase in the percentage of the population surviving into old age. This has increased the

Correspondence to: Mr. D. W. Flanagan, Department of Ophthalmology, Addenbrooke's Hospital, Hills Road, Cambridge CB2 2QQ, UK. number of older diabetics at risk of sight-threatening diabetic macular oedema. As a result there are now more diabetics with maculopathy than with proliferative retinopathy. Cataract surgery, particularly when done by the intra-capsular method in the presence of retinal ischaemia and oedema, frequently exacerbates diabetic retinopathy, making vision worse.

Effective screening followed by timely extensive scatter photocoagulation has changed this picture completely. Vitreoretinal surgery, though still essential in the minority of cases with advanced diabetic eye disease, is much less important. For example, in Addenbrooke's Hospital, Cambridge, in 1982 the bulk of vitreoretinal surgery was on diabetics with advanced diabetic eye disease. In that year almost 400 diabetics had vitreoretinal surgery. By 1989 this number had dropped to only 50, and has dropped further since.

If there is an effective screening service the majority of patients attending a diabetic eye clinic today will have one or more of these conditions: (1) moderate/severe peripheral retinal ischaemia, (2) macular oedema, (3) cataract and (4) posterior capsule opacification. Effective treatment depends on following these patients for long periods of time at appropriate intervals and offering treatment when vision is threatened. Vitreoretinal surgery should really only be needed occasionally for vitreous haemorrhage and rarely for traction retinal detachment. Cataract is extremely common in diabetics, as is posterior capsule opacification. Good cataract surgery with careful pre- and post-operative photocoagulation is as important as good vitreo-retinal surgery in the care of patients with established diabetic eye disease. Purchasing authorities in the future will look carefully at the outcome of treatment of all aspects of diabetic eye disease before they divert more resources to it.

\section{SEVERE PERIPHERAL RETINAL ISCHAEMIA AND RETINAL NEOVASCULARISATION}

The management of severe peripheral retinal ischaemia 


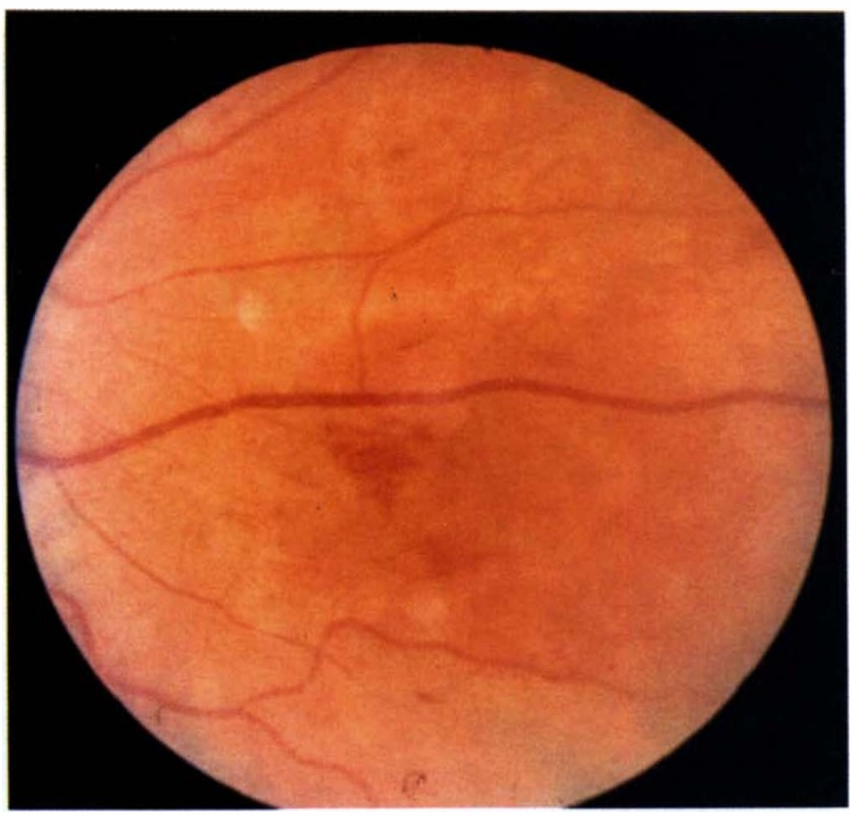

Fig. 1. Venous dilatation and slight beading indicating significant retinal ischaemia requiring close monitoring.

depends upon its early recognition, allowing timely photocoagulation of appropriate intensity with minimal complications.

Venous changes, retinal neovascularisation, retinal haemorrhages, macular oedema and cotton wool spots are the major indicators of severe retinal ischaemia. They determine the timing of photocoagulation and its intensity. These clinical signs also dictate how closely retinae should be monitored before and after photocoagulation. Finally, regression of these signs tells us that the retina is stable and that no further photocoagulation is required. In my experience cotton wool spots are of very limited value in predicting severe, irreversible peripheral retinal ischae-

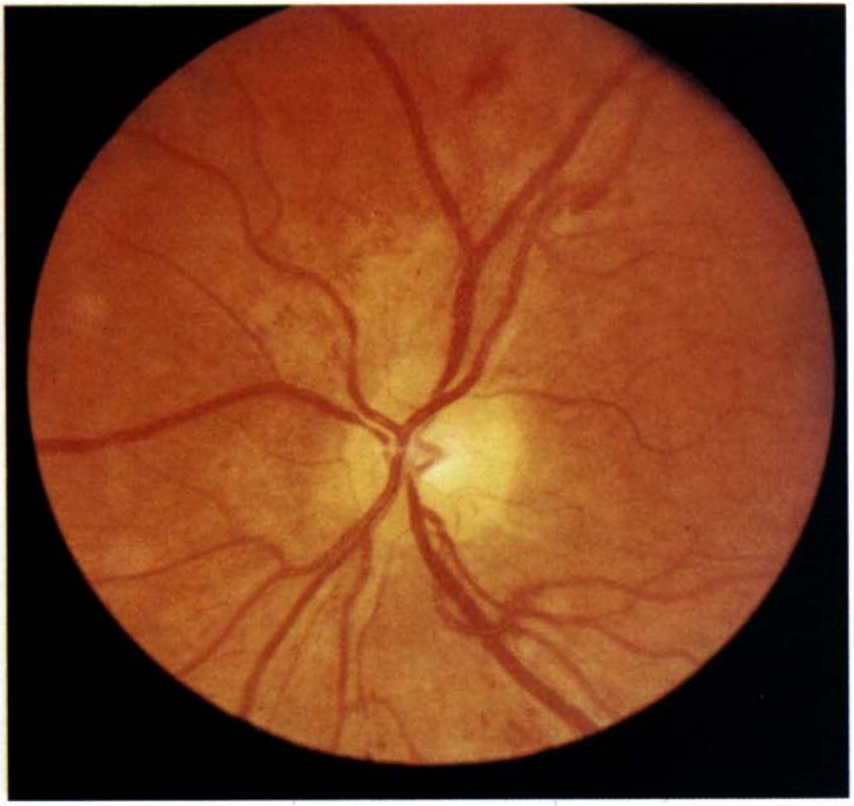

Fig. 3. Venous dilatation indicating significant retinal ischaemia requiring close monitoring.

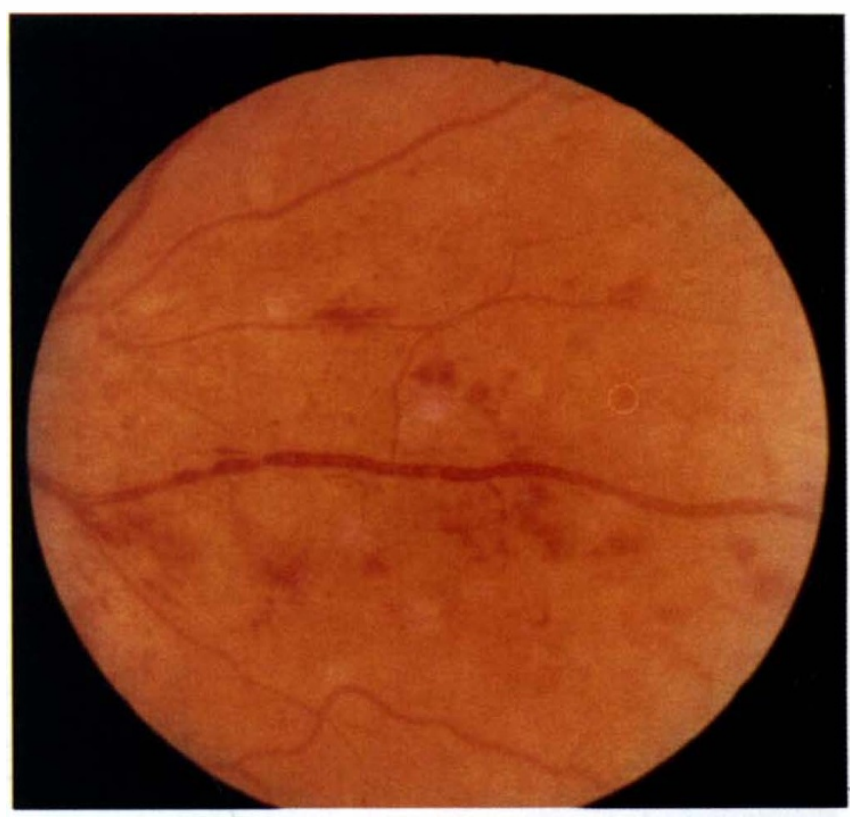

Fig. 2. Same eye as Fig. 1, 4 months later. Marked venous dilatation and beading indicate severe retinal ischaemia.

mia. They more commonly indicate co-existing uncontrolled hypertension or a recent dramatic improvement in blood sugar control.

In young insulin-dependent diabetics venous changes and macular oedema are the most important clinical signs of severe retinal ischaemia. The timing and intensity of treatment, as well as the frequency of follow-up, depend on these signs. There is a limit to the number of patients that can be seen, so follow-up visits must be kept to the minimum consistent with safety. Fig. 2 shows marked venous dilatation and beading, indicating severe peripheral retinal ischaemia with a high risk of neovascularisation if it is not already present. Such an eye obviously

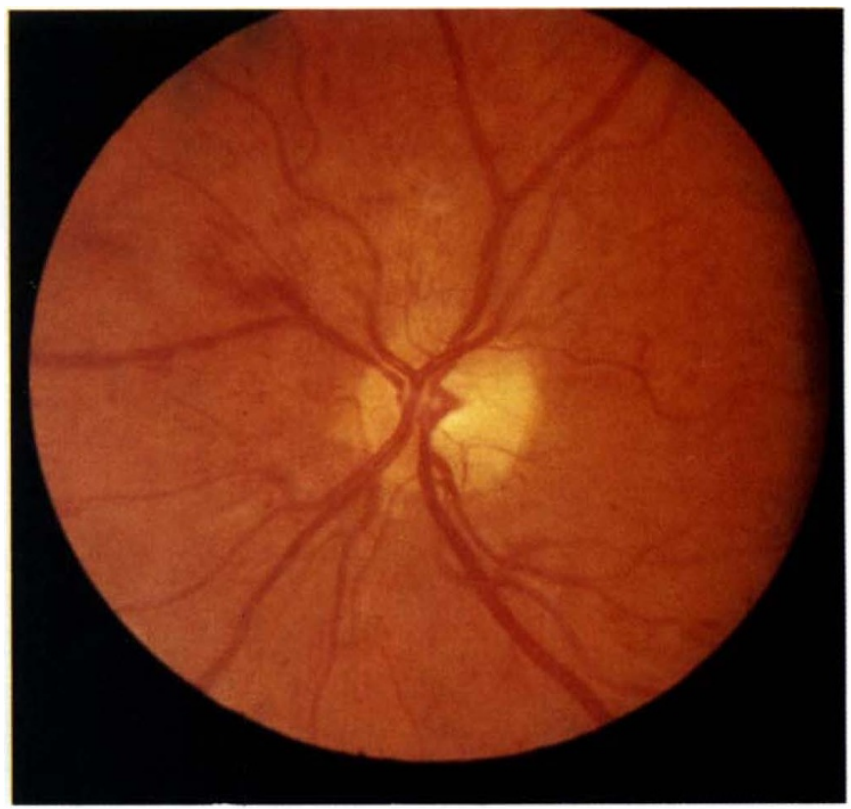

Fig. 4. Same eye as Fig. 3, with marked increase in venous dilatation and beading. 


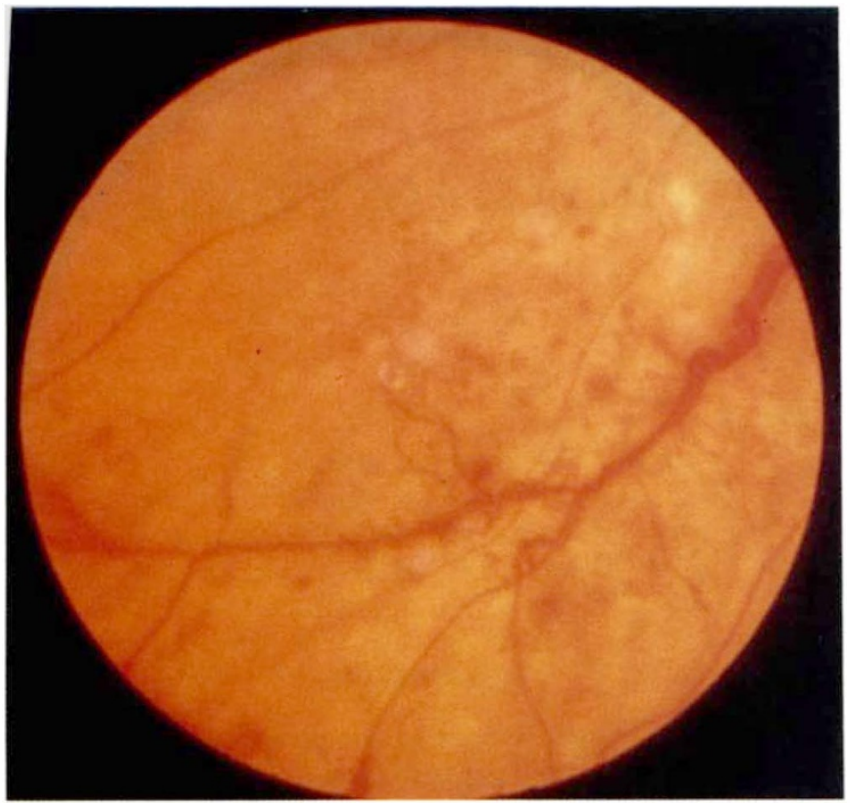

Fig. 5. Gross venous changes indicating extensive retinal ischaemia requiring early intense photocoagulation.

merits very close follow-up or probably immediate photocoagulation. Fig. 1 shows the same area of retina 4 months earlier. The venous changes are already present to a lesser degree. Early signs such as these are invaluable in detecting how closely to follow a patient at risk of posterior segment neovascularisation. Figs. 3 and 4 of the same eye demonstrate the change in venous characteristics over 4 months, indicating rapidly worsening retinal ischaemia and the need for close follow-up.

Retinal venous changes are also helpful in deciding how much photocoagulation is likely to be required. Eyes with very marked changes, such as in Fig. 5, need early intense photocoagulation if progression to retinal detachment is to

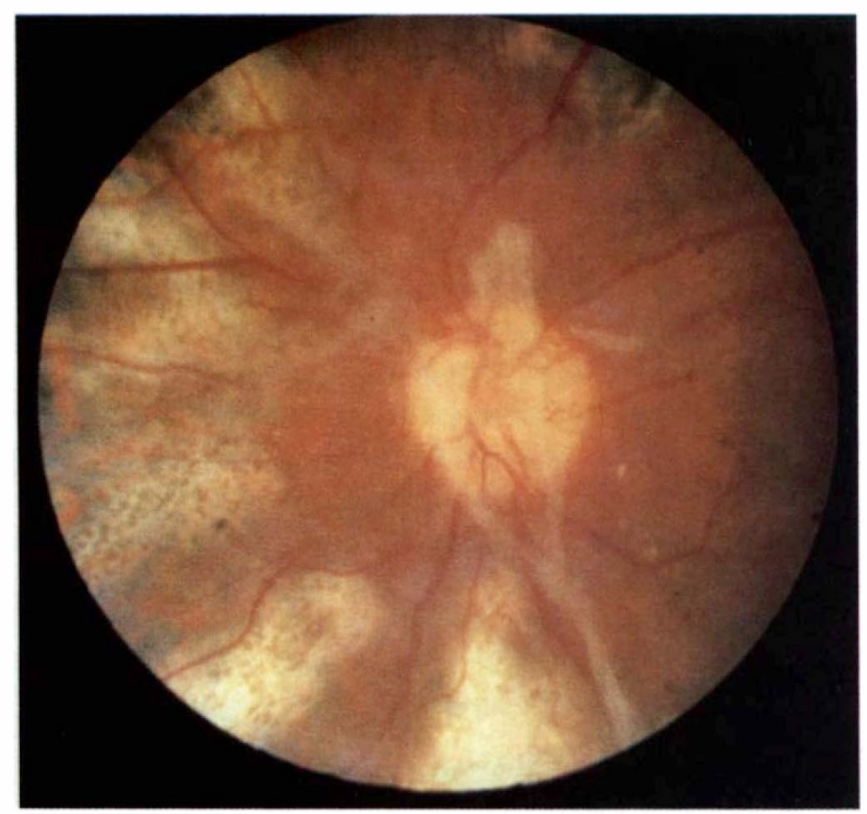

Fig. 7. The same eye as Fig. 6 after sufficient photocoagulation, showing normal-calibre veins.

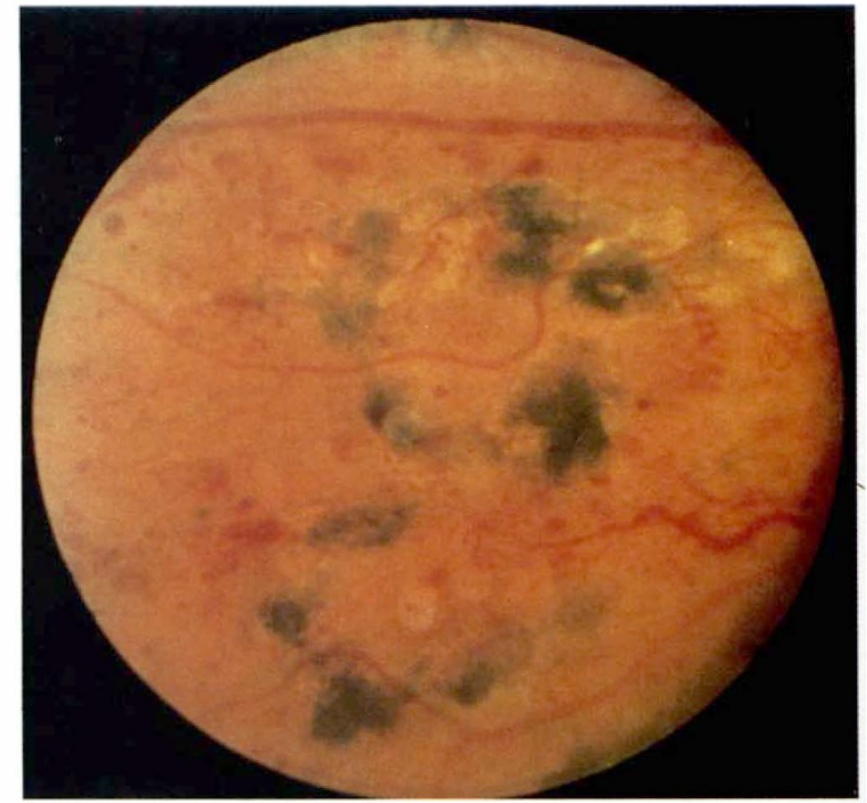

Fig. 6. Inadequate photocoagulation as shown by the persistent venous changes.

be avoided. In this case extreme venous dilatation is more useful in deciding how much photocoagulation is needed than the neovascularisation beside it.

The retina in Fig. 6 still has marked venous dilatation and beading and needs much more urgent photocoagulation. The same retina shown in Fig. 7 after further intensive photocoagulation has normal-calibre veins and is safe, requiring no further treatment. The retina in Fig. 8 has not required the same intense photocoagulation as that shown in Fig. 7, but no further photocoagulation is required as the veins have returned to normal calibre and the neovascularisation has disappeared.

Older maturity-onset diabetics do not always show the

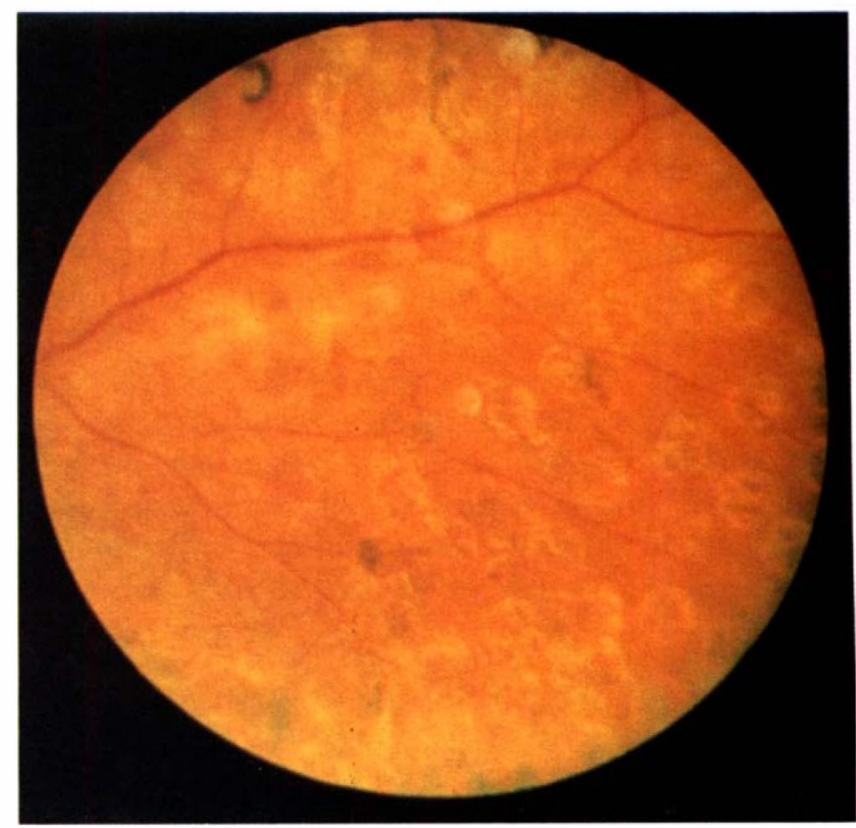

Fig. 8. Adequate gentle scatter photocoagulation with normal-calibre veins. 


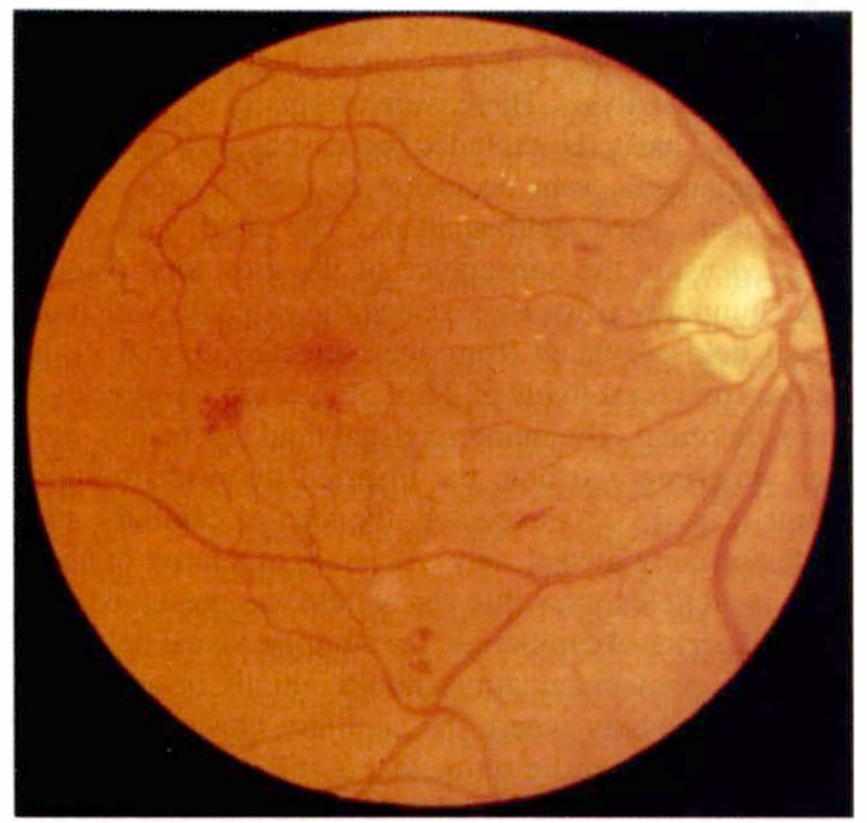

Fig. 9. 'Empty' retina: featureless, avascular and extremely ischaemic.

same dramatic vascular pre-proliferative signs of severe retinal ischaemia. The presence of a demarcation line between perfused posterior retina and ischaemic empty retina may be the only sign of severe retinal ischaemia. Red-free photographs frequently demonstrate this better than retinal colour photographs.

The retina in Fig. 9 shows none of the classical preproliferative signs yet 3 months later tufts of forward new vessels appeared as seen in Fig. 10. This peripheral retina is extremely ischaemic with a featureless, almost avascular, mid-peripheral retina.

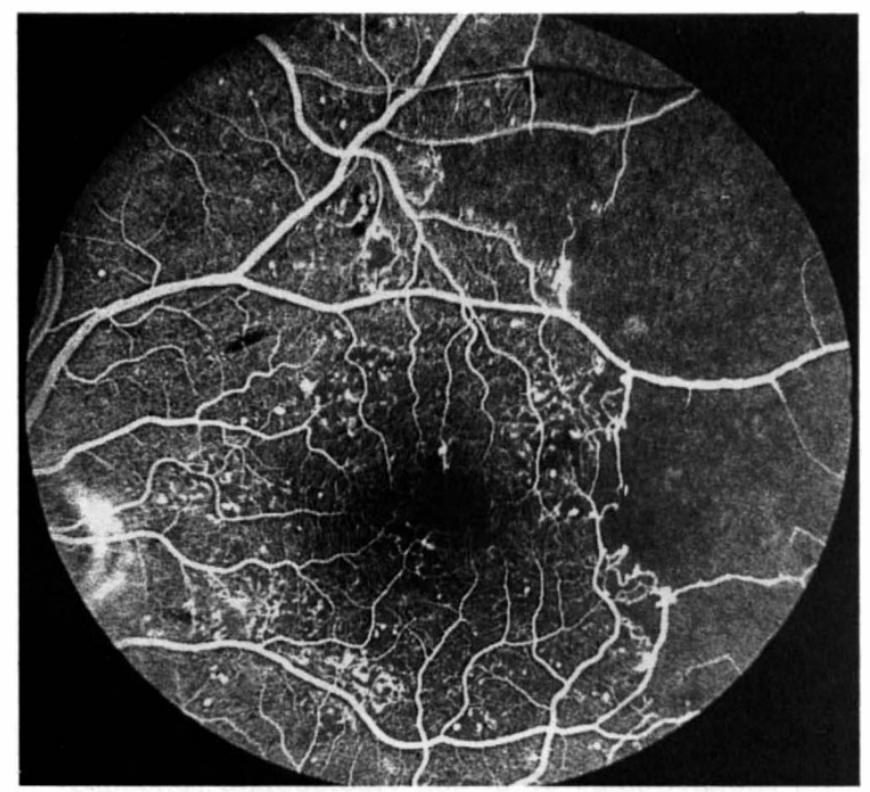

Fig. 11. Fluorescein angiogram showing extensive midperipheral retinal capillary closure.

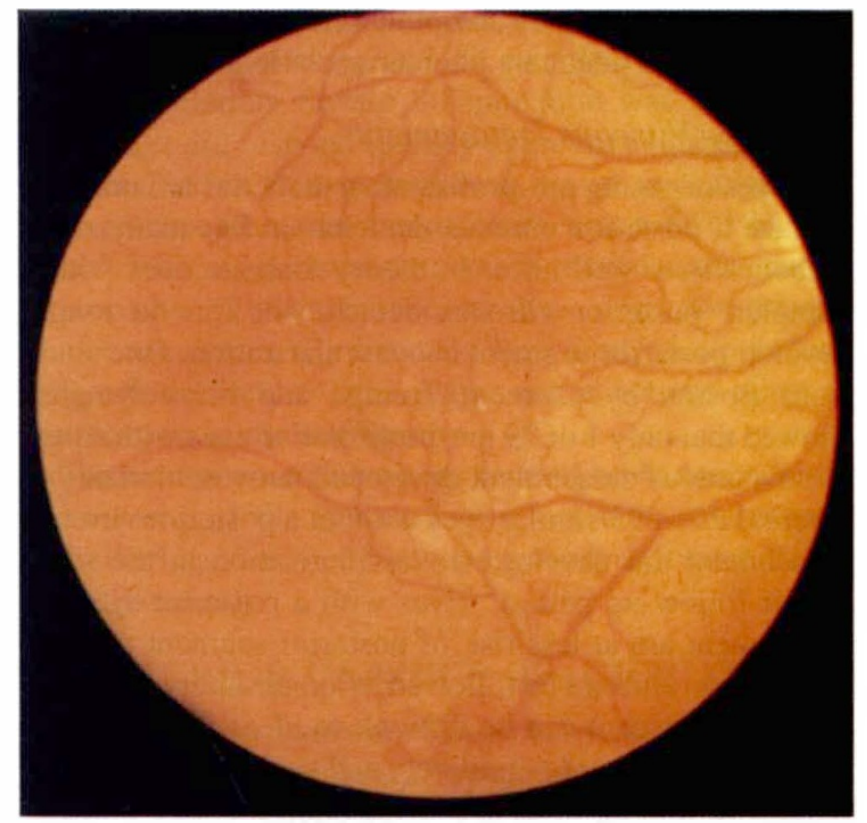

Fig. 10. The same eye as Fig. 9, 3 months later, with retinal neovascularisation superiorly.

\section{Long-Term Follow-up after Photocoagulation}

Many eye clinics now have to follow large numbers of relatively young healthy diabetics with stable retinae following extensive photocoagulation. Are such eyes safe from late visual loss? Do the patients require continued follow-up by an ophthalmologist or can they be discharged? Experience over 20 years by many observers suggests that such eyes are unlikely to have further problems but in theory visual loss from recurrent neovascularisation or macular oedema is possible. In 1990 George Blankenship in Miami reviewed 51 survivors of the original DRS Study of $1974 .{ }^{1}$ Only 2 of 51 eyes photocoagulated in 1974 had required additional photocoagulation in the 15 years after initial treatment. In his study the main cause of late visual loss appeared to be cataract. It

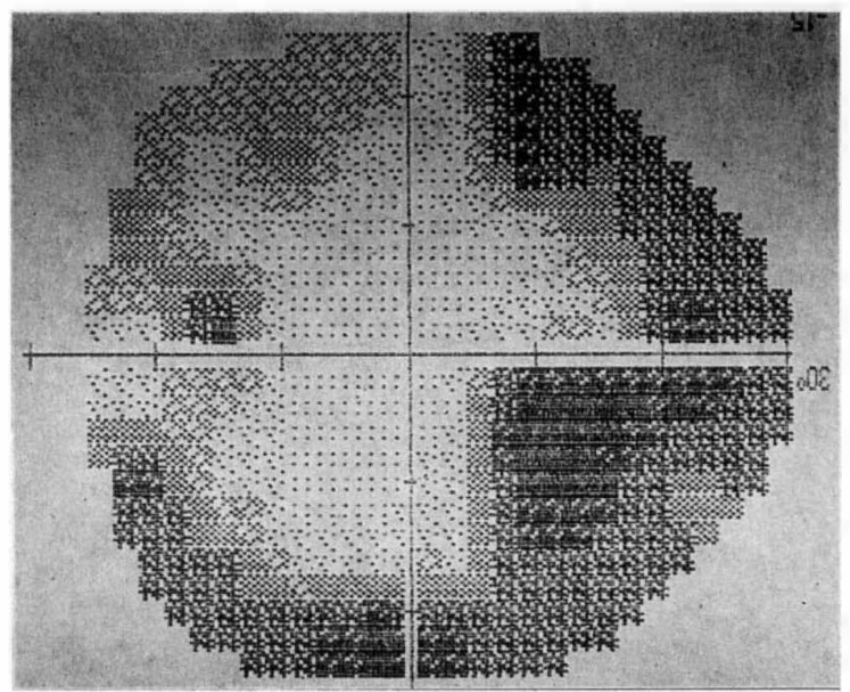

Fig. 12. Visual field loss corresponding to capillary closure in Fig. 11 . 
is possible, therefore, that young diabetics do not require long-term follow-up by an ophthalmologist once the eyes are stable after adequate photocoagulation.

\section{Posterior Vitreous Detachment}

The eyes of young pre-proliferative diabetics are unlikely to have a posterior vitreous detachment but many older diabetics may well have. In theory diabetic eyes with a complete posterior vitreous detachment can no longer develop posterior segment neovascularisation. One study from Boston by Clement Trempe and his colleagues showed that only 1 of 29 pre-proliferative eyes with a posterior vitreous detachment developed neovascularisation, whereas 20 of 93 similar eyes without a posterior vitreous detachment did develop neovascularisation in the same 3 -year follow-up period. ${ }^{2}$ Eyes with a posterior vitreous detachment are at less risk of posterior segment neovascularisation and can be followed at longer intervals. However, in practice it can be difficult to identify a posterior vitreous detachment definitively and such eyes frequently require follow-up in case they develop diabetic macular oedema requiring photocoagulation.

\section{Complications of Photocoagulation}

Photocoagulation, particularly with the xenon arc, can cause, or more frequently worsen, pre-existing visual field loss. ${ }^{3-7}$ Peripheral visual field constriction may mean that a diabetic can no longer keep his or her driving licence. ${ }^{8-10}$

Diabetic retinopathy before photocoagulation can cause visual field loss, as has been confirmed by many authors over the past 20 years. ${ }^{11-18}$ The field loss usually corresponds to areas of capillary non-perfusion and can be extensive. The fluorescein angiogram in Fig. 11 shows extensive mid-peripheral retinal closure with no neovascularisation as yet. The corresponding visual field in Fig. 12 shows extensive field loss.

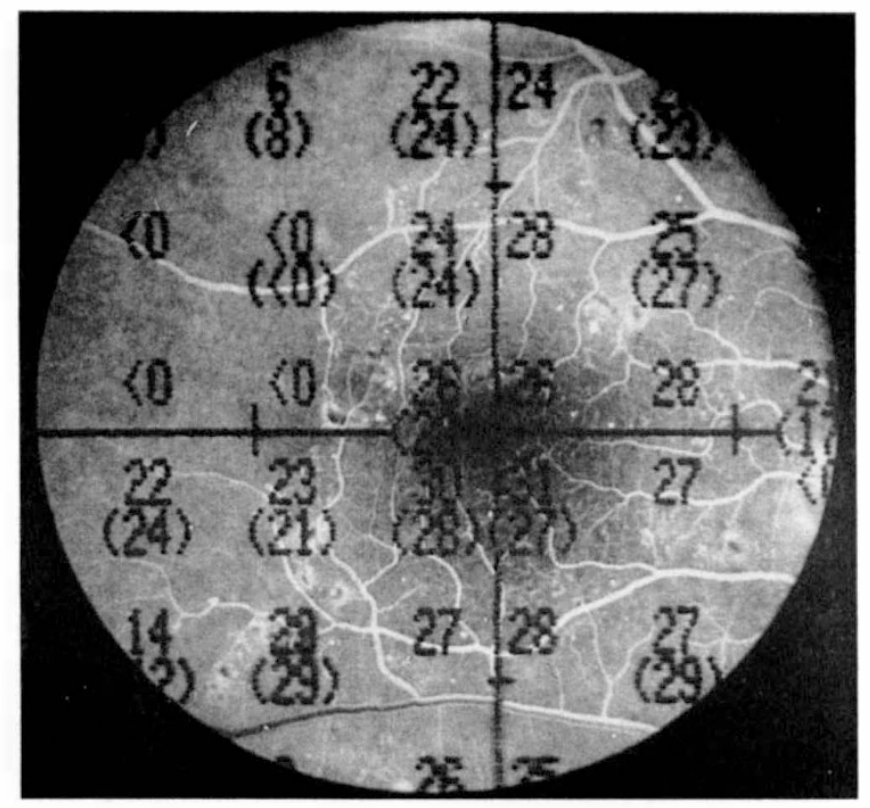

To discover the extent of visual field loss in pre-proliferative and early proliferative diabetics we looked at 30 eyes of 18 patients by fluorescein angiography and Humphrey 30-2 analysis. There were originally 37 eyes in the study, but 7 had to be excluded for reasons such as coexisting hemianopia, vein occlusion or inability to perform automated visual field analysis. The field defect was detected using the appearance of the grey tone display. The Humphrey software revealed the extent to which the visual fields deviated from age-corrected normal visual fields. The visual fields of 28 of 30 pre-proliferative eyes were abnormal. The numeric visual field test results were then superimposed onto the inverted fluorescein angiograms (Fig. 13). As expected, the area of capillary nonperfusion correlated closely with areas of relative or absolute visual field loss, while visual field was preserved over areas of perfused capillaries. To minimise visual field loss, therefore, photocoagulation should initially be applied to the mid-periphery where the capillary closure is worst, sparing more posterior retina that is still perfused. This is unlikely to worsen the visual field as the burns are applied to areas that have already lost visual field. In addition, directing the photocoagulation at the most ischaemic areas will maximise its effect. It should not be necessary initially to treat near the disc or just temporal to the macula. Blankenship has compared the results of peripheral and central panretinal photocoagulation. ${ }^{19}$ These showed that panretinal photocoagulation concentrated on the midperiphery appeared to be as effective as conventional or more posterior panretinal photocoagulation. There was, however, less field loss and less loss of acuity from macular oedema with peripheral panretinal photocoagulation . This approach may, however, result in inadequate photocoagulation. Frequent follow-up by experienced observers is therefore mandatory until the retina is completely stable.

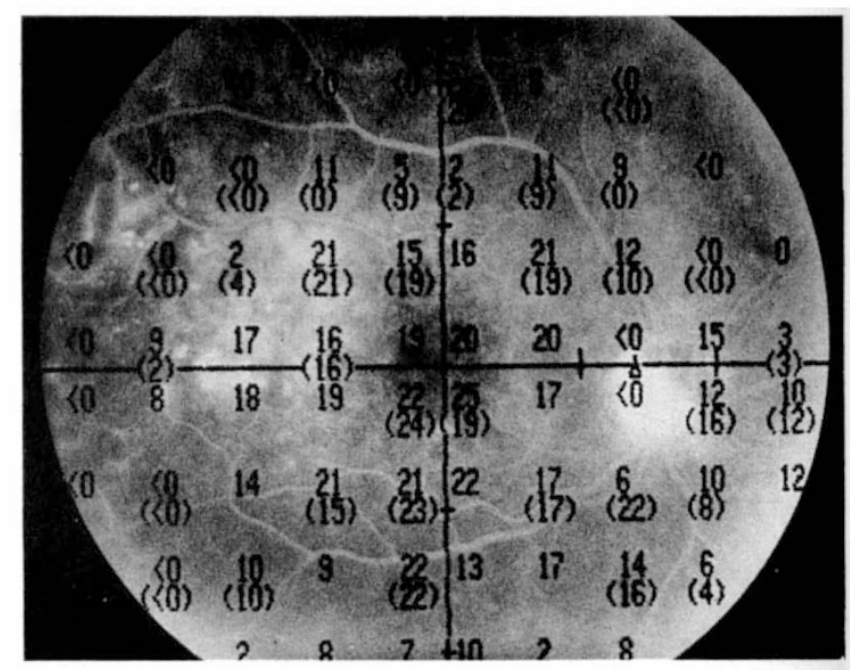

Fig. 13. Threshold visual field results superimposed on corresponding fluorescein angiograms, showing correlation between capillary closure and visual field loss. 


\section{DIABETIC MACULAR OEDEMA}

Sight-threatening diabetic macular oedema is largely, but not exclusively, a problem of older, non-insulin-dependent diabetic patients. The prevalence of non-insulindependent diabetes mellitus has grown as the proportion of the population surviving into old age has increased. Visual loss due to diabetic maculopathy is now a greater problem numerically than visual loss from proliferative diabetic retinopathy. Screening non-insulin-dependent diabetic patients for maculopathy can be difficult for nonophthalmologists due to coexisting cataract and poor miosis. The recently completed Early Treatment Diabetic Retinopathy Study confirms that photocoagulation is most effective if applied before there is significant foveal oedema or central visual loss. Physicians with noninsulin-dependent diabetic patients in their care therefore need to refer to ophthalmologists if they detect any significant diabetic maculopathy. This policy is liable to increase the workload in eye clinics. However, it is probably the only way to ensure that diabetic maculopathy is treated at the optimum time. ${ }^{20}$

\section{CATARACT AND DIABETIC RETINOPATHY}

Cataract is common in diabetics. ${ }^{21.22}$ Figures from Addenbrooke's Hospital, Cambridge, show that $9.5 \%$ of all insulin-dependent diabetics either have cataracts or have had cataract surgery. The corresponding figure for noninsulin-dependent diabetics was $12.3 \%$.

For a long time there was understandable reluctance to insert intraocular lenses into diabetic eyes: $;^{23-26}$ this was in case it made assessment of retinopathy difficult or interfered with photocoagulation. In addition, there have been a number of reports of a dramatic exacerbation of macular oedema with visual loss following cataract surgery and intraocular lens insertion. ${ }^{27}$ To see just how well diabetics did after cataract and intraocular lens surgery we compared the visual results of cataract surgery in a group of diabetics with those in a group of non-diabetic agematched controls. ${ }^{28}$ After exclusions there were 66 diabetics with 66 matching controls. Thirty-three diabetics had no retinopathy pre-operatively while 23 had background (11 with maculopathy) and 10 had proliferative retinopathy (8 with coexisting maculopathy).

Overall, visual acuity improved in 57 eyes, was unchanged in 4 eyes and deteriorated in 5 of the 66 eyes. Fewer diabetics than controls achieved a visual acuity of $6 / 12$. Significantly more diabetics than controls did badly, with a visual acuity of less than $6 / 60$. However, when eyes with maculopathy were excluded, diabetics did as well as control eyes, with $85 \%$ achieving $6 / 12$ or better. Most of the eyes which had $6 / 36$ or $6 / 60$ vision had maculopathy. Vision improved in 6 of 10 eyes with proliferative diabetic retinopathy, remained unchanged in 2 and deteriorated in 2 . The 5 eyes in which vision deteriorated all had preexisting maculopathy.

Diabetics undoubtedly had more complications postoperatively, particularly macular oedema, severe inflammation and capsular thickening. Four eyes developed rubeosis and neovascular glaucoma, 2 after YAG capsulotomy. However, it was possible to photocoagulate successfully in most cases and 17 eyes had post-operative photocoagulation. Severe inflammation was more common but it did respond to early aggressive treatment.

Overall, 57 of 66 eyes improved, 4 were unchanged and 5 were worse after a minimum follow-up period of 2 years. In conclusion: (1) visual outcome is related to the state of retinopathy, (2) the intraocular lens does not prevent photocoagulation, (3) diabetics have more post-operative inflammation and (4) the major complications relate to progression of the retinopathy.

The results would have been better if we had recognised the severity of the ischaemia and macular oedema preoperatively and treated it more aggressively either pre- or post-operatively.

\section{POSTERIOR CAPSULE OPACIFICATION}

Two eyes in our study developed neovascular glaucoma after a YAG capsulotomy. Presumably cutting the posterior capsule encourages iris neovascularisation by allowing vasoproliferative factors to leak forwards. It is important, therefore, to assess diabetic eyes carefully following YAG capsulotomy, if necessary using fluorescein angiography. If there is significant peripheral retinal ischaemia such eyes should have photocoagulation either before or shortly after YAG capsulotomy.

In summary, eyes with established diabetic eye disease tend to have one or more of these four conditions: (1) peripheral retinal ischaemia, (2) macular oedema, (3) cataract and (4) posterior capsule opacification.

Most diabetics with established diabetic eye disease can maintain reasonable, but not perfect, vision provided they are followed carefully and offered timely appropriate treatment. Vitreoretinal surgery should only be required in the few patients who refuse screening or who develop persistent vitreous haemorrhage before photocoagulation is completed. Progression to blindness from vitreous haemorrhage, traction retinal detachment, neovascular glaucoma or macular oedema should be exceptional.

I would like to thank Katherine Haslam, Medical Photographer, for the retinal photographs, and Dr. Caroline Chee, FRCS, for the visual field analysis.

\section{REFERENCES}

1. Blankenship GW. Fifteen year argon laser and xenon photocoagulation results of Bascom Palmer Eye Institute's patients participating in the diabetic retinopathy study. Ophthalmology 1991;98:125-8.

2. Akiba J, Carlos WA, Trempe CL. Posterior vitreous detachment and neovascularisation in diabetic retinopathy. Ophthalmology 1990;97:889-91.

3. Trick GL, Trick LR, Kilo C. Visual field defects in patients with insulin-dependent and non-insulin-dependent diabetes. Ophthalmology 1990;97:475-82.

4. Bell JA, Feldon SE. Retinal microangiopathy: correlation of OCTOPUS perimetry with fluorescein angiography. Arch Ophthalmol 1984;102:1294-8.

5. Hamilton AM, Townsend C, Khoury D, Gould E, Blach RK. Xenon arc and argon laser photocoagulation in the treatment 
of diabetic disc neovascularisation. I. Effect on disc vessels, visual field and visual acuity. Trans Ophthalmol Soc UK 1981;101:87-92.

6. Blankenship GW. A clinical comparison of central and peripheral argon laser panretinal photocoagulation for proliferative diabetic retinopathy. Ophthalmology 1988;95:170-7.

7. Chee CKL, Flanagan DW. Visual field loss in preproliferative and early proliferative diabetic retinopathy. Br J Ophthalmol (submitted).

8. Williamson TH, George N, Flanagan DW, Norris V, Blamires T. Driving standard visual fields in diabetic patients after panretinal laser photocoagulation. In: Vision in Vehicles III. Amsterdam: North-Holland, 1991:265-72.

9. Hulbert MFG, Vernon SA. Passing the DVLC field regulations following bilateral pan-retinal photocoagulation in diabetics. Eye 1992;6:456-60.

10. Buckley SA, Jenkins L, Benjamin L. Fields, DVLC and panretinal photocoagulation. Eye 1992;6:623-5.

11. Roth JA. Central visual field in diabetes. Br J Ophthalmol 1969;53:16-25.

12. Caird FI, Pirie A, Ramsell IG. Diabetes and the eye. Oxford: Blackwell Scientific, 1969.

13. Taylor E, Dobree JH. Proliferative diabetic retinopathy: site and size of initial lesions. Br J Ophthalmol 1970;54:11-8.

14. Wiznia KI, Lieberman TW, Leopold IH. Visual fields in diabetic retinopathy. Br J Ophthalmol 1970;54:11-8.

15. Greite JH, Zumbansen HP, Adamczyk R. Visual field in diabetic retinopathy (DR). In: Greve EL, Verriest G, editors. Fourth International Visual Field Symposium, Bristol, 13-16 April 1980. Documenta Ophthalmologica Proceedings Series 26. The Hague: Junk, 1981:25-32.

16. Beck T. Coexistence of localised scotomata and neovascularisations in proliferative diabetic retinopathy. Acta Ophthalmol (Copenh) 1990;68:421-7.

17. Beck T, Lund-Anderson H. Localised blood-retinal barrier leakage and retinal light sensitivity in diabetic retinopathy. Br J Ophthalmol 1990;74:388-92.

18. Beck T, Lund-Anderson H. Cotton-wool spots and retinal light sensitivity in diabetic retinopathy. $\mathrm{Br} \mathrm{J}$ Ophthalmol 1991;75:13-7.

19. Blankenship GW. A clinical comparison of central and peripheral argon laser panretinal photocoagulation for proliferative diabetic retinopathy. Ophthalmology 1988;95: 170-7.

20. ETDRS Report Number 9. Early photocoagulation for diabetic retinopathy. Ophthalmology 1991;98:766-85.

21. Klein BEK, Klein R, Moss SE. Prevalence of cataracts in a population-based study of persons with diabetes mellitus. Ophthalmology 1985;92:1191-6.

22. Straatsma BR, Pettit TH, Wheeler N, Miyamasu W. Diabetes mellitus and intraocular lens implantation. Ophthalmology 1983;90:336-43.

23. Thompson SM, Kritzinger EE, Roper-Hall MJ. Should diabetes be a contraindication for an intraocular lens? Trans Ophthalmol Soc UK 1983;103:115-7.

24. Patz A. Photocoagulation of retinal, vascular and macular diseases through intraocular lenses. Ophthalmology 1981; 88:398-406.

25. Jaffe NS. The way things were and are: changing indications for intraocular lens implantation. Ophthalmology 1983;90: 318-20.

26. Tasman W. Are there any retinal contraindications to cataract extraction and posterior chamber lens implants? Arch Ophthalmol 1986;104:1767-8.

27. Jaffe GJ, Burton TC. Progression of nonproliferative diabetic retinopathy following cataract extraction. Arch Ophthalmol 1988;106:745-9.

28. Cunliffe IA, Flanagan DW, George NDL, Aggarwaal RJ, Moore AT. Extracapsular cataract surgery with lens implantation in diabetics with and without proliferative retinopathy. Br J Ophthalmol 1991;75:9-12. 